PROCEEDINGS OF THE

AMERICAN MATHEMATICAL SOCIETY

Volume 139, Number 11, November 2011, Pages 3937-3949

S 0002-9939(2011)10826-8

Article electronically published on March 11, 2011

\title{
PROPERTIES OF SOLUTIONS OF A CLASS OF PLANAR ELLIPTIC OPERATORS WITH DEGENERACIES
}

\author{
P. L. DATTORI DA SILVA AND A. MEZIANI
}

(Communicated by Mei-Chi Shaw)

\begin{abstract}
In this paper we investigate properties of solutions of first and second order elliptic equations that degenerate along a simple closed curve in $\mathbb{R}^{2}$. These equations are generated by a $\mathbb{C}$-valued vector field $L$. To the vector field $L$, we associate the second order operator $\mathbb{P}=\operatorname{Re}[L \bar{L}+p L]$, where $p$ is a $\mathbb{C}$-valued function. We establish a one-to-one correspondence between the solutions of the equation $\mathbb{P} u=0$ and those of an associated first order equation of type $L w=A w+B \bar{w}$.
\end{abstract}

\section{INTRODUCTION}

In this paper we investigate some properties of the solutions of first and second order elliptic equations that degenerate along a simple closed curve in $\mathbb{R}^{2}$. These equations are generated by a $\mathbb{C}$-valued vector field $L$. We assume that $L$ is elliptic everywhere except along a simple closed curve on which it is tangent and $L \wedge \bar{L}$ vanishes to an order $>1$. The main equation considered here is

$$
L w=A w+B \bar{w} .
$$

The case when the order of vanishing of $L \wedge \bar{L}$ is one is considered by the second author in 7, 8, and 10. The techniques in the above papers will be generalized to the case studied here. The approach and motivations for the study of such

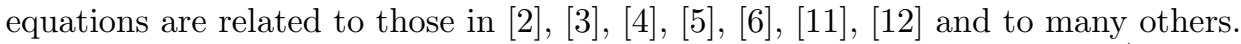

For equation (1.1), we establish the existence of a set of basic solutions $w_{j}^{ \pm}, j \in \mathbb{Z}$. This set plays an important role in the structure of the space of solutions of (1.1). This system is complete, and every solution $w$ of (1.1) has a series representation in the $w_{j}^{ \pm}$'s and is similar to one of the basic solutions. This means that for every solution $w$ of (1.1) there is $j_{0} \in \mathbb{Z}$ and a nonvanishing function $P$ such that $w=w_{j_{0}}^{ \pm} P$. A uniqueness result for the solutions of (1.1) also holds. More precisely, if $w$ solves (1.1) in an open set $U$ with $\Sigma \subset U$ and if $u$ vanishes on a set of points in $U \backslash \Sigma$ that accumulates on $\Sigma$, then $w=0$ on the connected component of $U \backslash \Sigma$ that contains the sequence of points.

To the vector field $L$, we associate the second order operator

$$
\mathbb{P}=\operatorname{Re}[L \bar{L}+p L]
$$

Received by the editors April 22, 2010 and, in revised form, September 9, 2010. 2010 Mathematics Subject Classification. Primary 35C10; Secondary 35F05.

Key words and phrases. Elliptic equations, series representation, normalization.

The first author was supported in part by CNPq and FAPESP.

(C)2011 American Mathematical Society Reverts to public domain 28 years from publication 
where $p$ is a $\mathbb{C}$-valued function. As was done in [9] and in [10, we establish a one-to-one correspondence between the solutions of the equation $\mathbb{P} u=0$ and those of an associated first order equation of type (1.1). To each solution $u$, we associate its $L$-gradient $w$, a solution of an equation (1.1); and vice versa, to each solution $w$, we can associate its $L$-potential that satisfies $\mathbb{P} u=0$. This correspondence allows us to deduce properties of the second order equation from those of the first order.

The organization of this paper is as follows. In section 2 , we prove that under suitable conditions, the vector field $L$ is integrable in a neighborhood of the characteristic set $\Sigma$. In section 3, properties of the solutions of the first order equation are established. In sections 4 and 5, we reduce the study of the second order equation into the first order and deduce the properties of its solutions.

\section{Normalization of $L$}

In this section, we prove a normalization of the vector field $L$ in a full neighborhood of the characteristic set $\Sigma$.

Without loss of generality, we can assume that $\Sigma=\{0\} \times \mathbb{S}^{1}$ is a circle in $\mathbb{R} \times \mathbb{S}^{1}$ so that $L$ is a $\mathbb{C}$-valued vector field of class $C^{k}$ (with $k \geq 1$ ) in a cylinder $A_{\epsilon}=(-\epsilon, \epsilon) \times \mathbb{S}^{1}$ (with $\epsilon>0$ ). We assume that $L$ is elliptic in $A_{\epsilon} \backslash \Sigma$ and that it is tangent to $\Sigma$ at each point $p \in \Sigma$. Thus, we can assume (after multiplying $L$ by a nonvanishing function) that $L$ is given in the coordinates $(r, t)$ of $\mathbb{R} \times \mathbb{S}^{1}$ by

$$
L=\frac{\partial}{\partial t}-i(C(r, t)+i D(r, t)) \frac{\partial}{\partial r}
$$

where $C, D \in C^{k}\left(A_{\epsilon}, \mathbb{R}\right), C(r, t) \neq 0$ for every $r \neq 0$, and $C(0, t)=D(0, t)=0$ for every $t \in \mathbb{S}^{1}$. Throughout this paper, we assume that there exists a function $\alpha \in C^{k}((-\epsilon, \epsilon), \mathbb{R})$ such that $\alpha(0)=\alpha^{\prime}(0)=0, \alpha(r) \neq 0$ for $r \neq 0$ and such that:

$\left(H_{1}\right)$ The coefficients of $L$ satisfy $C(r, t)+i D(r, t)=\alpha(r)\left(C_{1}(r, t)+i D_{1}(r, t)\right)$ with $C_{1}(0, t) \neq 0, D_{1}(0, t)=0, t \in \mathbb{S}^{1}$.

$\left(H_{2}\right)$ A primitive $F(r)$ of the function $\frac{1}{\alpha(r)}$ satisfies

$$
\lim _{r \rightarrow 0}|F(r)|=\infty \text {. }
$$

Examples of such functions $\alpha$ include $r^{1+\mu}$ with $\mu>0$ and $r / \ln |r|$. Hence, from now on, we will assume that

$$
L=\frac{\partial}{\partial t}-i \alpha(r)[P(r, t)+i Q(r, t)] \frac{\partial}{\partial r}
$$

with $P, Q$ real-valued, $P(0, t)>0$ and $Q(0, t)=0$ for all $t \in \mathbb{S}^{1}$. Throughout this paper we will use the notation $A_{\epsilon}^{+}=(0, \epsilon) \times \mathbb{S}^{1}$ and $A_{\epsilon}^{-}=(-\epsilon, 0) \times \mathbb{S}^{1}$. We have the following integrability for the vector field $L$.

Theorem 1. Let $L$ be a vector field given by (2.1) whose coefficients satisfy conditions $\left(H_{1}\right)$ and $\left(H_{2}\right)$. Then there exist $\epsilon>0$ and diffeomorphisms

$$
\Phi^{ \pm}: \overline{A_{\epsilon}^{ \pm}} \longrightarrow \Phi^{ \pm}\left(\overline{A_{\epsilon}^{ \pm}}\right) \text {with } \Phi^{ \pm}(\Sigma)=\Sigma
$$

and such that the pushforward $\Phi_{*}^{ \pm} L$ is a multiple of the vector field

$$
T_{\alpha}=\frac{\partial}{\partial t}-i \alpha(r) \frac{\partial}{\partial r}
$$


Proof. Let $\nu=\frac{1}{2 \pi} \int_{0}^{2 \pi} P(0, t) d t$. We then have

$$
P(0, t)=\nu\left(1+P_{1}(t)\right) \quad \text { with } \quad \int_{0}^{2 \pi} P_{1}(t) d t=0,
$$

and it follows from the hypothesis on $L$ that we can assume that $\nu>0$ and $1+$ $P_{1}(t)>0$ for every $t$. In fact, after factoring out $\nu$ and replacing $\alpha(r)$ by $\nu \alpha(r)$, we can assume that $\nu=1$. Hence,

$$
L=\frac{\partial}{\partial t}-i \alpha(r)\left[1+P_{1}(t)+S(r, t)\right] \frac{\partial}{\partial r},
$$

with $S(r, t)$ a $\mathbb{C}$-valued, continuous function satisfying $S(0, t)=0$. Define a new angle $\phi$ on $\mathbb{S}^{1}$ by

$$
\phi=t+\int_{0}^{t} P_{1}(s) d s .
$$

Note that $\phi(t)$ is an increasing function and $\phi(t+2 \pi)=\phi(t)+2 \pi$. With respect to the coordinates $(r, \phi)$, defined near circle $r=0$ in $\mathbb{R} \times \mathbb{S}^{1}$, the vector field $L$ becomes a multiple of the vector field

$$
L_{1}=\frac{\partial}{\partial \phi}-i \alpha(r)[1+M(r, \phi)] \frac{\partial}{\partial r} \quad \text { with } \quad M(r, \phi)=\frac{S(r, \phi)}{1+P_{1}(\phi)} .
$$

Let $F(r)$ be a primitive of the function $1 / \alpha(r)$ satisfying condition $\left(H_{2}\right)$. Assume first that

$$
\lim _{r \rightarrow 0^{+}} F(r)=-\infty \text {. }
$$

Let $\rho=\exp (F(r))$. Then $\rho \rightarrow 0^{+}$as $r \rightarrow 0^{+}$and $\rho:(0, \epsilon) \longrightarrow(0, \exp (F(\epsilon)))$ is a diffeomorphism. With respect to the coordinates $(\rho, \phi)$, the expression of $L_{1}$ becomes

$$
L_{1}=\frac{\partial}{\partial \phi}-i \rho(1+\hat{S}(\rho, \phi)) \frac{\partial}{\partial \rho},
$$

with $\hat{S}$ continuous up to the circle $\rho=0$ and $\hat{S}(0, \phi)=0$.

Let $Z=\rho \mathrm{e}^{i \phi}$. The pushforward via $Z$ of $L_{1}$ is the vector field defined in a neighborhood of $0 \in \mathbb{C}$ by

$$
Z_{*} L_{1}=-i \bar{Z}(2+\hat{S}(Z))\left[\frac{\partial}{\partial \bar{Z}}+\mu(Z) \frac{\partial}{\partial Z}\right]
$$

with

$$
\mu(Z)=\frac{\hat{S}(Z)}{2+\hat{S}(Z)} \frac{Z}{\bar{Z}}=\frac{\hat{S}(\rho, \phi)}{2+\hat{S}(\rho, \phi)} \mathrm{e}^{2 i \phi} .
$$

Note that since $\mu(Z)$ is continuous and $\mu(0)=0$, it follows from the general theory of the Beltrami equation (see [1] or [13]) that the equation

$$
\frac{\partial W}{\partial \bar{Z}}+\mu(Z) \frac{\partial W}{\partial Z}=0
$$

has a nontrivial solution $W \in C^{1, \sigma}$ defined in a neighborhood of $0 \in \mathbb{C}$ and moreover $W$ can be taken of the form

$$
W(Z)=Z(1+G(Z))
$$

with $G(0)=0$. Consider the coordinates $\left(r^{*}, t^{*}\right)$ in a cylinder $A_{\epsilon}^{+}$(with $\epsilon$ small) given by

$$
r^{*}=F^{-1}(\log |W(Z)|), \quad t^{*}=\arg (W(Z)) .
$$


We then have $W=\exp \left(F\left(r^{*}\right)\right) \mathrm{e}^{i t^{*}}$, and it follows that in these coordinates the vector field $L_{1}$ and consequently $L$ is a multiple of

$$
\frac{\partial}{\partial t^{*}}-i \alpha\left(r^{*}\right) \frac{\partial}{\partial r^{*}}
$$

The theorem is then proved in this case.

For the case when $\lim _{r \rightarrow 0^{+}} F(r)=+\infty$, we consider $\rho=\exp (-F(r))$ and the function $Z=\rho \mathrm{e}^{-i \phi}$. With this adjustment, the rest of the proof follows in a similar way.

As a consequence of this normalization, we get that any continuous solution of the homogeneous equation $L u=0$ is flat along the circle $\Sigma$. By a flat function along $\Sigma$, we mean a function $u(r, t)$ such that $u(0, t)=u_{0}$ is constant and

$$
\lim _{r \rightarrow 0} \frac{u(r, t)-u_{0}}{r^{N}}=0, \quad \forall N>0 .
$$

Theorem 2. Suppose that in addition to the above properties, $\alpha \in C^{1, \tau}$ for some $0<\tau<1$. Then, if $u$ is a continuous solution of $L u=0$ in a tubular neighborhood of $\Sigma$, then $u$ is flat along $\Sigma$.

Proof. It follows from the hypotheses $\alpha \in C^{1, \tau}$ and $\alpha(0)=\alpha^{\prime}(0)=0$ that $|\alpha(r)|<$ $C|r|^{1+\tau}$ for some positive constant $C$ and for $|r|<\epsilon_{0}$. Assume that $\alpha(r)>0$ for $r>0(\alpha(r)<0$ for $r>0$ is analogous $)$. Then

$$
F(r)=\int_{\epsilon_{0}}^{r} \frac{d s}{\alpha(s)}<-\frac{K}{r^{\tau}} \quad r \in\left(0, \frac{\epsilon_{0}}{2}\right),
$$

for some positive constant $K$. Hence, the function $\rho(r)=\exp (F(r)) \leq \exp \left(-K / r^{\tau}\right)$ is flat at 0 . Consequently, the first integral $Z(r, \phi)=\rho(r) \mathrm{e}^{i \phi}$ is flat along the circle $\Sigma$. Now if $u$ is continuous on $\left[0, \epsilon_{0}\right) \times \mathbb{S}^{1}$ and solves $L u=0$, then $u$ can be written as $u(r, \phi)=H \circ Z(r, \phi)$ for some holomorphic function $H$ in the disc $D(0,1)$. Thus $u$ is flat along the circle $\Sigma$. A similar argument can be used in the cylinder $A_{\epsilon_{0}}^{-}$.

Corollary 3. Suppose that the coefficient $\alpha$ is of class $C^{\infty}$ and satisfies the following property: there exist constants $\epsilon_{0}>0, C>0$, and $\sigma>0$ such that

$$
\left|\int_{r}^{ \pm \epsilon} \frac{d s}{\alpha(s)}\right| \geq \frac{C}{|\alpha(r)|^{\sigma}}
$$

If $u$ is a continuous solution of $L u=0$ in a tubular neighborhood of $\Sigma$, then $u \in$ $C^{\infty}\left(A_{\epsilon}\right)$ for some $\epsilon>0$.

Proof. Assume that $\alpha(r)>0$ in $A_{\epsilon_{0}}^{+}$. Then it follows from (2.3) that $F(r)=$ $\int_{\epsilon_{0}}^{r} \frac{d s}{\alpha(s)}$ satisfies $F(r) \leq-C /(\alpha(r))^{\sigma}$ for $0<r<\epsilon_{0}$. Furthermore, the function $\exp (F(r))$ is $C^{\infty}$ on the interval $\left[0, \epsilon_{0}\right)$ and vanishes to infinite order at 0 . It follows that the first integral $Z$ is $C^{\infty}\left(\left[0, \epsilon_{0}\right) \times \mathbb{S}^{1}\right)$ and vanishes to infinite order along the circle $\Sigma$. Now, if $u$ is bounded in $A_{\epsilon_{0}}^{+}$and solves $L u=0$, then as in the proof of Theorem 2, $u=H \circ Z$, with $H$ holomorphic. Consequently, $u$ is $C^{\infty}$ and is flat along $\Sigma$. A similar argument can be used in the cylinder $A_{\epsilon_{0}}^{-}$to complete the proof. 


\section{The model homogeneous equation $T_{\alpha} w=b \bar{w}$}

In this section, we consider the first order equation for the vector field $L$. Thanks to the normalization theorem of section 2 , we can assume that the vector field is given by expression (2.2) in the cylinder $A_{\epsilon}=(-\epsilon, \epsilon) \times \mathbb{S}^{1}$. That is, we consider

$$
T_{\alpha}=\frac{\partial}{\partial t}-i \alpha(r) \frac{\partial}{\partial r},
$$

where the $\mathbb{R}$-valued function $\alpha$ is of class $C^{1}, \alpha(0)=\alpha^{\prime}(0)=0, \alpha(r) \neq 0$ for $r \neq 0$, and $\alpha$ satisfies condition $\left(H_{2}\right)$. Thus, there is a function $F(r)$ defined for $r \neq 0$ and such that

$$
F^{\prime}(r)=\frac{1}{\alpha(r)}, \quad \lim _{r \rightarrow 0}|F(r)|=\infty .
$$

We will assume without loss of generality that $\lim _{r \rightarrow 0^{+}} F(r)=-\infty$.

The model equation for the vector field is

$$
T_{\alpha} w=b(t) \bar{w}
$$

where $b \in C^{k}\left(\mathbb{S}^{1}, \mathbb{C}\right)$.

Remark 4. The more general equation $T_{\alpha} w=A(t) w+B(t) \bar{w}$ with $\int_{0}^{2 \pi} A(t) d t \in$ $2 \pi \mathbb{Z}$ can be reduced to an equation of the form (3.2). Indeed, if $w$ solves $T_{\alpha} w=$ $A w+B \bar{w}$, then the function $w_{1}=\exp \left(-\int_{0}^{t} A(s) d s\right) w$ solves equation (3.2) with $b(t)=B(t) \exp \left(-2 i \int_{0}^{t} \operatorname{Im}(A(s)) d s\right)$.

Theorem 5. Let $b \in C^{k}\left(\mathbb{S}^{1} ; \mathbb{C}\right)$, with $k \geq 2$. There exist a sequence of real numbers $\lambda_{j}^{ \pm}$and a sequence of functions $\psi_{j}^{ \pm} \in C^{k+1}\left(\mathbb{S}^{1} ; \mathbb{C}\right)$ such that for every $j \in \mathbb{Z}$ the functions

$$
w_{j}^{ \pm}(r, t)=e^{\lambda_{j}^{ \pm} F(r)} \psi_{j}^{ \pm}(t)
$$

solve equation (3.2) in the cylinder $A_{\epsilon} \backslash \Sigma$. Moreover, the sequence $\left(\lambda_{j}^{ \pm}\right)$satisfies

$$
\ldots<\lambda_{-1}^{-} \leq \lambda_{-1}^{+}<\lambda_{0}^{-} \leq \lambda_{0}^{+}<\lambda_{1}^{-} \leq \lambda_{1}^{+}<\ldots,
$$

with

$$
\lim _{j \rightarrow-\infty} \lambda_{j}^{ \pm}=-\infty \quad \text { and } \quad \lim _{j \rightarrow \infty} \lambda_{j}^{ \pm}=\infty
$$

and

$$
\int_{0}^{2 \pi} \arg \left(\psi_{j}^{ \pm}(\theta)\right) d \theta=2 \pi j
$$

Proof. We use the first integral $\Phi(r, t)=\exp (F(r)) \mathrm{e}^{i t}$ of $T_{\alpha}$ in $A_{\epsilon} \backslash \Sigma$ to transform equation (3.2) in $A_{\epsilon}^{+}=A_{\epsilon} \cap\{r>0\}$ and in $A_{\epsilon}^{-}=A_{\epsilon} \cap\{r<0\}$ into CR equations. Indeed, the pushforward of (3.2) in $A_{\epsilon}^{ \pm}$is the CR equation

$$
\frac{\partial U_{ \pm}}{\partial \bar{z}}=i \frac{b(t)}{2 \bar{z}} \overline{U_{ \pm}} .
$$

We know from 8 that such a CR equation has solutions of the form

$$
U_{ \pm, j}^{ \pm}(z)=|z|^{\lambda_{j}^{ \pm}} \psi_{j}^{ \pm}(\arg (z))
$$


where the $\lambda_{j}^{ \pm}$'s and the $\psi_{j}^{ \pm}$'s satisfy the conditions of the theorem. The functions

$$
w_{j}^{ \pm}(r, t)=U_{ \pm, j}^{ \pm}(\Phi(r, t))=\exp \left(\lambda_{j}^{ \pm} F(r)\right) \psi_{j}^{ \pm}(t)
$$

are therefore solutions of (3.2) with the desired properties.

Remark 6 . The $\lambda_{j}^{ \pm}$and $\psi_{j}^{ \pm}$are, respectively, the eigenvalues and eigenfunctions of the periodic ode

$$
\psi^{\prime}(t)-i \lambda \psi(t)=b(t) \overline{\psi(t)} .
$$

If for an eigenvalue $\lambda_{j}$ the ode has two independent eigenfunctions $\psi_{j}^{+}$and $\psi_{j}^{-}$, then $\lambda_{j}^{-}=\lambda_{j}^{+}$. When the equation has only one eigenfunction, then $\lambda_{j}^{-}<\lambda_{j}^{+}$.

Let $L_{\mathbb{R}}^{2}\left(\mathbb{S}^{1} ; \mathbb{C}\right)$ be the space of $\mathbb{C}$-valued, $L^{2}$-functions on $\mathbb{S}^{1}$, which is considered as an $\mathbb{R}$-vector space and is equipped with the inner product given by

$$
\langle\varphi, \psi\rangle=\frac{1}{2 \pi} \int_{0}^{2 \pi} \operatorname{Re}(\varphi(\theta) \bar{\psi}(\theta)) d \theta .
$$

It follows from $\left[\underline{8}\right.$ that the system $\left\{\psi_{j}^{ \pm}\right\}_{j \in \mathbb{Z}}$ is complete in $L_{\mathbb{R}}^{2}\left(S^{1} ; \mathbb{C}\right)$ and can be taken to be orthogonal. The asymptotic behavior $($ as $|j| \rightarrow \infty)$ of the $\lambda_{j}^{ \pm}$and $\psi_{j}^{ \pm}$ is given by

$$
\begin{aligned}
& \lambda_{j}^{+}=\lambda_{j}^{-}+O\left(\frac{1}{j^{2}}\right)=j+\frac{\gamma}{2 j}+O\left(\frac{1}{j^{2}}\right), \\
& \psi_{j}^{+}(t)=\mathrm{e}^{i\left(j t+m_{j}\right)}+O\left(\frac{1}{j}\right) \text { and } \psi_{j}^{-}(t)=i \mathrm{e}^{i\left(j t+m_{j}\right)}+O\left(\frac{1}{j}\right),
\end{aligned}
$$

where $\gamma=\frac{1}{2 \pi} \int_{0}^{2 \pi}|b(t)|^{2} d t$ and with $m_{j} \in[0, \pi)$.

An immediate consequence is that every function $g \in L_{\mathbb{R}}^{2}\left(S^{1} ; \mathbb{C}\right)$ has a Fourier series representation

$$
g(t)=\sum_{j \in \mathbb{Z}} g_{j}^{+} \psi_{j}^{+}(t)+g_{j}^{-} \psi_{j}^{-}(t)
$$

where $g_{j}^{ \pm}=\left\langle g, \psi_{j}^{ \pm}\right\rangle$. Moreover, if $g \in C^{k}$, then $g_{j}^{ \pm}=O\left(1 / j^{k}\right)$. This allows us to obtain a series representation for the solutions of $(3.2)$.

Theorem 7. Let $w(r, t)$ be a solution of $(3.2)$ continuous on $\overline{A_{\epsilon}^{+}}$. Then there exist sequences of real numbers $c_{j}^{+}$and $c_{j}^{-}$such that

$$
w(r, t)=\sum_{\lambda_{j}^{ \pm} \geq 0}\left(c_{j}^{+} w_{j}^{+}(r, t)+c_{j}^{-} w_{j}^{-}(r, t)\right)
$$

where $w_{j}^{ \pm}(r, t)=e^{\lambda_{j}^{ \pm} F(r)} \psi_{j}^{ \pm}(t)$. Furthermore,

$$
\left|c_{j}^{ \pm}\right|=O(\exp (-j F(\epsilon))), \quad \text { as } \quad j \longrightarrow \infty .
$$

A similar representation holds if $w$ is a solution in $A_{\epsilon}^{-}$.

Proof. Let $w \in C^{0}\left(\overline{A_{\epsilon}^{+}}\right)$be a solution of (3.2). We use the $\left\{\psi_{j}^{ \pm}\right\}$-expansion to write $w$ as $w(r, t)=\sum_{j \in \mathbb{Z}} f_{j}^{ \pm}(r) \psi_{j}^{ \pm}(t)$, with $f_{j}^{ \pm}=\left\langle w, \psi_{j}^{ \pm}\right\rangle$. Then it follows from (3.2) and 
(3.9) that the coefficients $f_{j}^{ \pm}$satisfy the differential equation

$$
\alpha(r) \frac{d f_{j}^{ \pm}}{d r}=\lambda_{j}^{ \pm} f_{j}^{ \pm}
$$

Hence, $f_{j}^{ \pm}(r)=c_{j}^{ \pm} \exp \left(\lambda_{j}^{ \pm} F(r)\right)$, with $c_{j}^{ \pm} \in \mathbb{R}$. This establishes expansion (3.10). Furthermore, $c_{j}^{ \pm}=0$ for $\lambda_{j}^{ \pm}<0$ (otherwise $w$ would not be bounded). Since $w$ is continuous, then there exists $M>0$ such that

$$
\sup _{0<r<\epsilon}\left|c_{j}^{ \pm} \exp \left(\lambda_{j}^{ \pm} F(r)\right)\right| \leq M \quad \forall j \in \mathbb{Z} .
$$

Estimate (3.11) follows from the asymptotic expansion of the $\lambda_{j}^{ \pm}$.

As a consequence of this representation, we have the following uniqueness result.

Theorem 8. Let $w \in C^{0}\left(\overline{A_{\epsilon}^{+}}\right)$be a solution of (3.2). If there exists a sequence of points $\left(r_{k}, t_{k}\right) \in A_{\epsilon}^{+}$with $r_{k} \rightarrow 0$ and $w\left(r_{k}, t_{k}\right)=0$, then $w=0$ in $A_{\epsilon}^{+}$. An analogous result holds in $A_{\epsilon}^{-}$.

Proof. By using Theorem 7, we need to show that if $w(r, t)=\sum_{\lambda_{j}^{ \pm} \geq 0} c_{j}^{ \pm} w_{j}^{ \pm}(r, t)$ vanishes on the sequence $\left(r_{k}, t_{k}\right)$, then $c_{j}^{ \pm}=0$ for all $j \in \mathbb{Z}$. By contradiction, suppose that $w \neq 0$ and let $j_{0}$ be the smallest integer such that $c_{j_{0}}^{+} \neq 0$ or $c_{j_{0}}^{-} \neq 0$. Then, it follows from $w\left(r_{k}, t_{k}\right)=0$ and the series expansion that

$$
\begin{aligned}
c_{j_{0}}^{-} \psi_{j_{0}}^{-}\left(t_{k}\right) & +c_{j_{0}}^{+} \exp \left[\left(\lambda_{j_{0}}^{+}-\lambda_{j_{0}}^{-}\right) F\left(r_{k}\right)\right] \psi_{j_{0}}^{+}\left(t_{k}\right) \\
& +\sum_{j>j_{0}} c_{j}^{ \pm} \exp \left[\left(\lambda_{j}^{ \pm}-\lambda_{j_{0}}^{-}\right) F\left(r_{k}\right)\right] \psi_{j}^{ \pm}\left(t_{k}\right)=0 .
\end{aligned}
$$

We can assume that the sequence $r_{k}$ is decreasing and that $t_{k} \rightarrow t_{0}$. It follows from $F(r)$ decreasing to $-\infty$ that when we let $k \rightarrow \infty$, we obtain

$$
\begin{array}{rll}
c_{j_{0}}^{-} \psi_{j_{0}}^{-}\left(t_{0}\right)=0 & \text { when } \quad \lambda_{j_{0}}^{-}<\lambda_{j_{0}}^{+}, \\
c_{j_{0}}^{-} \psi_{j_{0}}^{-}\left(t_{0}\right)+c_{j_{0}}^{+} \psi_{j_{0}}^{+}\left(t_{0}\right)=0 & \text { when } \quad \lambda_{j_{0}}^{-}=\lambda_{j_{0}}^{+} .
\end{array}
$$

Since $\psi_{j_{0}}^{ \pm}$are nontrivial solutions of the ode (3.9) and are independent (when the ode has all solutions periodic), then necessarily $c_{j_{0}}^{-}=0$ in the first case and $c_{j_{0}}^{-}=c_{j 0}^{+}=0$ in the second case. This completes the proof.

For the nonhomogeneous equation

$$
T_{\alpha} w=b(t) \bar{w}+G(r, t)
$$

we have the following result.

Theorem 9. Let $G(r, t)=\alpha(r) g(r, t)$, where $g \in C^{2}\left(\overline{A_{\epsilon}^{+}}\right)$. Then equation (3.12) has a solution $w \in C^{0}\left(\overline{A_{\epsilon}^{+}}\right)$.

Proof. We use the $\left\{\psi_{j}^{ \pm}\right\}$-expansions

$$
w(r, t)=\sum_{j \in \mathbb{Z}} w_{j}^{ \pm}(r) \psi_{j}^{ \pm}(t) \quad \text { and } \quad G(r, t)=\sum_{j \in \mathbb{Z}} G_{j}^{ \pm}(r) \psi_{j}^{ \pm}(t) .
$$


Note that $G_{j}^{ \pm}(r)=\alpha(r) g_{j}^{ \pm}(r)$. It follows at once from (3.9) that if $w$ is a solution of (3.12), then the function $w_{j}^{ \pm}$satisfies the differential equation

$$
\alpha(r) \frac{d w_{j}^{ \pm}}{d r}-\lambda_{j}^{ \pm} w_{j}^{ \pm}=i G_{j}^{ \pm}(r) .
$$

Hence, we can define

$$
w_{j}^{ \pm}(r)=-\int_{r}^{\epsilon} i g_{j}^{ \pm}(s) e^{\lambda_{j}^{ \pm}(F(r)-F(s))} d s, \quad \text { if } \quad \lambda_{j}^{ \pm} \geq 0,
$$

and

$$
w_{j}^{ \pm}(r)=\int_{0}^{r} i g_{j}^{ \pm}(s) e^{\lambda_{j}^{ \pm}(F(r)-F(s))} d s, \quad \text { if } \quad \lambda_{j}^{ \pm}<0 .
$$

Note that the sign of $\lambda_{j}^{ \pm}$in the formulaes (3.14) and (3.15) implies that $\lambda_{j}^{ \pm}(F(r)-$ $F(s)) \leq 0$ in both situations.

Finally, the fact that $g \in C^{2}$ implies in particular that

$$
\max _{0 \leq r \leq \epsilon}\left|j g_{j}^{ \pm}(r)\right| \rightarrow 0, \quad \text { as } \quad j \rightarrow \pm \infty,
$$

and, consequently, also

$$
\max _{0 \leq r \leq \epsilon}\left|j w_{j}^{ \pm}(r)\right| \rightarrow 0, \quad \text { as } \quad j \rightarrow \pm \infty .
$$

Therefore, $w(r, t)=\sum_{j \in \mathbb{Z}} w_{j}^{ \pm}(r) \psi_{j}^{ \pm}(t) \in C^{0}\left(\overline{A_{\epsilon}^{+}}\right)$, where $w_{j}^{ \pm}$is given by (3.14) and (3.15), is a solution for (3.12).

\section{Reduction of SECOND order EQUation into A FiRst order}

We show here that properties of a second order equation can be understood in terms of those of an associated first order model equation (3.2). Consider the operator $P$ defined as

$$
P=T_{\alpha} \overline{T_{\alpha}}+a(t) T_{\alpha}+\bar{a}(t) \overline{T_{\alpha}},
$$

where $T_{\alpha}$ is the vector field given in (3.1) and with $a \in C^{k}\left(S^{1}\right), k \geq 1$, satisfying

$$
\frac{1}{2 \pi i} \int_{0}^{2 \pi} a(t) d t \in \mathbb{Z}
$$

To the equation $P u=0$ we are going to associate an equation of type (3.2). For this, we define the function

$$
A(t)=\exp \int_{0}^{t} \bar{a}(s) d s
$$

which is a $2 \pi$-periodic function of class $C^{k+1}$. Note that $T_{\alpha} A=\bar{a} A$. Define

$$
b(t)=-a(t) \frac{A(t)}{\bar{A}(t)}
$$

We have

$$
T_{\alpha} A=\bar{a} A=\frac{\bar{a} \bar{A} A^{2}}{A \bar{A}}=-\bar{b} \frac{A^{2}}{\bar{A}} .
$$

To each real-valued function $u$, we associate the complex-valued function $w$ defined by

$$
w(r, t)=A(t) \overline{T_{\alpha}} u(r, t) .
$$


We will refer to $w$ as the $T_{\alpha}$-gradient of $u$ with respect to $P$. To the operator $P$ we associate the first order operator $\mathcal{L}$ defined by

$$
\mathcal{L} w=T_{\alpha} w-b(t) \bar{w}
$$

where $b(t)$ is given by (4.2). Before we proceed further, we will need a Green's identity for the operator $\mathcal{L}$.

For $\mathbb{C}$-valued functions defined on an open set $\mathcal{U} \subset \mathbb{R}_{+} \times S^{1}$, we define the bilinear form

We have

$$
\langle f, g\rangle=\operatorname{Re}\left(\iint_{\mathcal{U}} f(r, t) g(r, t) \frac{d r d t}{\alpha(r)}\right) .
$$

$$
\langle\mathcal{L} f, g\rangle=\operatorname{Re}\left(\iint_{\mathcal{U}}(\mathcal{L} f) g \frac{d r d t}{\alpha(r)}\right)=\operatorname{Re}\left(\iint_{\mathcal{U}} f\left(-\frac{\partial g}{\partial t}+i \alpha(r) \frac{\partial g}{\partial r}-\overline{b g}\right) \frac{d r d t}{\alpha(r)}\right) .
$$

Hence, the adjoint operator $\mathcal{L}^{*}$ is given by

$$
\mathcal{L}^{*} g=-\left(T_{\alpha} g+\overline{b g}\right) \text {. }
$$

As before, we will make use of the first integral $Z(r, t)=\exp (F(r)) \mathrm{e}^{i t}$ to obtain Green's identity.

Lemma 10. Let $U \subset A_{\epsilon}^{+}$be an open set with piecewise smooth boundary. Let $u, v \in C^{0}(\bar{U})$ with $\mathcal{L} u$ and $\mathcal{L} v$ integrable. Then

$$
\operatorname{Re}\left(\int_{\partial U} u v \frac{d z}{z}\right)=\left\langle u, \mathcal{L}^{*} v\right\rangle-\langle\mathcal{L} u, v\rangle
$$

Proof. A simple calculation shows that for a differentiable function $f(r, t)$ we have

$$
d f=\frac{i}{2}\left(-\overline{T_{\alpha}} f \frac{d z}{z}+T_{\alpha} f \frac{d \bar{z}}{\bar{z}}\right)
$$

Moreover,

$$
\frac{d z}{z} \wedge \frac{d \bar{z}}{\bar{z}}=\frac{2 i}{\alpha(r)} d r \wedge d t
$$

Hence,

$$
\begin{aligned}
\operatorname{Re}\left(\int_{\partial U} u v \frac{d z}{z}\right) & =\operatorname{Re}\left(\iint_{U} \frac{i}{2}\left(-\overline{T_{\alpha}}(u v) \frac{d z}{z}+T_{\alpha}(u v) \frac{d \bar{z}}{\bar{z}}\right) \frac{d z}{z}\right) \\
& =\operatorname{Re}\left(\iint_{U} \frac{i}{2}\left(u T_{\alpha} v+v T_{\alpha} u\right) \frac{d \bar{z}}{\bar{z}} \wedge \frac{d z}{z}\right) \\
& =\operatorname{Re}\left(\iint_{U} \frac{i}{2}\left(u T_{\alpha} v+v T_{\alpha} u-c \bar{u} v+\bar{c} u \bar{v}\right) \frac{2 i}{\alpha(r)} d r d t\right) \\
& =\left\langle u, \mathcal{L}^{*} v\right\rangle-\langle\mathcal{L} u, v\rangle .
\end{aligned}
$$

In the next proposition, we reduce the study of the equation for $P$ into an equation for $T_{\alpha}$.

Proposition 11. Suppose that $G$ is a real-valued function and that $u(r, t)$ is realvalued and solves the equation

$$
P u(r, t)=G(r, t)
$$

in the cylinder $A_{\epsilon}^{+}$. Then its $T_{\alpha}$-gradient $w$ satisfies

$$
\mathcal{L} w=A(t) G(r, t) .
$$


Conversely, if $w$ is a solution of (4.8) in $A_{\epsilon}^{+}$, then there exists a real-valued function $u$ defined in $A_{\epsilon}^{+}$that solves (4.7) and whose $T_{\alpha}$-gradient is $w$. More precisely,

$$
u(r, t)=\operatorname{Re} \int_{\left(r_{0}, t_{0}\right)}^{(r, t)} \frac{w(\rho, \theta)}{A(\theta)} \frac{d \zeta}{i \zeta}
$$

where $\zeta=\exp (F(\rho)) \mathrm{e}^{i \theta}$ and the integration is taken over any simple curve in $A_{\epsilon}^{+}$ that joins the fixed point $\left(r_{0}, t_{0}\right)$ to the point $(r, t)$.

Proof. Suppose that $u$ is real-valued and solves (4.7). Let $w$ be the $T_{\alpha}$-gradient of $u$. We have

$$
\begin{aligned}
T_{\alpha} w & =T_{\alpha}\left(A \overline{T_{\alpha}} u\right)=T_{\alpha} A \overline{T_{\alpha}} u+A T_{\alpha} \overline{T_{\alpha}} u \\
& =\bar{a} A \overline{T_{\alpha}} u+A\left(G-a T_{\alpha} u-\bar{a} \overline{T_{\alpha}} u\right)=A G+b(t) \bar{w},
\end{aligned}
$$

which implies that $w$ solves (4.8).

Conversely, suppose that $w$ solves (4.8). Let $\left(r_{0}, t_{0}\right) \in A_{\epsilon}^{+}$and consider $u$ defined by (4.9). First, we have to show that $u$ is well-defined. That is, we have to show that the integral is path independent. Let $U$ be a relatively compact subset of $A_{\epsilon}^{+}$whose boundary consists of simple closed curves. It follows from the proof of Green's identity (4.6) and (4.5) that

$$
\begin{aligned}
\int_{\partial U} \frac{w(\zeta)}{A(\theta)} \frac{d \zeta}{i \zeta} & =\frac{1}{2} \iint_{U} T_{\alpha}\left(\frac{w}{A}\right) \frac{d \bar{\zeta}}{\bar{\zeta}} \wedge \frac{d \zeta}{\zeta}=i \iint_{U} T_{\alpha}\left(\frac{w}{A}\right) \frac{d \rho d \theta}{\alpha(\rho)} \\
& =i \iint_{U}\left(\frac{T_{\alpha} w}{A}-w \frac{T_{\alpha} A}{A^{2}}\right) \frac{d \rho d \theta}{\alpha(\rho)}=i \iint_{U}\left(\frac{T_{\alpha} w}{A}+w \frac{\bar{b}}{\bar{A}}\right) \frac{d \rho d \theta}{\alpha(\rho)} \\
& =i \iint_{U}\left(G+\bar{w} \frac{b}{A}+w \frac{\bar{b}}{\bar{A}}\right) \frac{d \rho d \theta}{\alpha(\rho)}
\end{aligned}
$$

Since $G$ is real-valued, this implies that

$$
\operatorname{Re} \int_{\partial U} \frac{w(\rho, \theta)}{A(\theta)} \frac{d \zeta}{i \zeta}=0
$$

therefore, $u$ is well-defined.

Finally, we will show that $u$ defined by (4.9) is a solution for (4.7). We have

$$
\frac{\partial u}{\partial t}=\operatorname{Re}\left(\frac{w}{A}\right) \quad \text { and } \quad \frac{\partial u}{\partial r}=\frac{1}{\alpha(r)} \operatorname{Im}\left(\frac{w}{A}\right) ;
$$

hence, $\overline{T_{\alpha}} u=\frac{w}{A}$. Now, simple calculations show that $P u=G$.

\section{The homogeneous equation $P u=0$}

In this section we will use the reduction given by Proposition 11 to obtain properties of the solutions of the equation $P u=0$ from those of their $T_{\alpha}$-gradients $w$. We start with the following.

Remark 12. If $u \equiv u(t)$ is a solution to $P u=0$, then $u$ is constant. This is immediate from the periodic ordinary differential equation $u^{\prime \prime}(t)+2 \operatorname{Re}(a(t)) u^{\prime}(t)=$ 0 . 
Lemma 13. Let $u(r, t)$ be a real-valued function. Assume that $u$ is a solution of $P u=0$ in the cylinder $A_{\epsilon}^{+}$and let $w=A \overline{T_{\alpha}} u$ be its $T_{\alpha}$-gradient. If

$$
\operatorname{Re}\left(\frac{w(r, t)}{i A(t)}\right) \equiv 0,
$$

then $u$ is constant.

Proof. Let $\left(r_{0}, 0\right)$ be a fixed point in the cylinder $A_{\epsilon}^{+}$. Let $u$ be as in the statement and let $w$ be its $T_{\alpha}$-gradient. Define

$$
v(r, t)=\operatorname{Re} \int_{\Gamma(r, t)} \frac{w(\zeta)}{A(\theta)} \frac{d \zeta}{i \zeta},
$$

where $\Gamma(r, t)$ is any piecewise smooth curve that joins the point $\left(r_{0}, 0\right)$ to the point $(r, t)$. It follows from Proposition 11 that $P v=0$. If we choose $\Gamma(r, t)=\Gamma_{1} \cup \Gamma_{2}$, where

$$
\Gamma_{1}=\left\{\left(r_{0}, s t\right) ; 0 \leq s \leq 1\right\} \quad \text { and } \quad \Gamma_{2}=\left\{\left((1-s) r_{0}+s r, t\right) ; 0 \leq s \leq 1\right\},
$$

we have

$$
\int_{\Gamma_{1}} \frac{w(\zeta)}{A(\theta)} \frac{d \zeta}{i \zeta}=t \int_{0}^{1} \frac{w\left(r_{0}, s t\right)}{A(s t)} d s
$$

and

$$
\int_{\Gamma_{2}} \frac{w(\zeta)}{A(\theta)} \frac{d \zeta}{i \zeta}=\int_{0}^{1} \frac{w\left((1-s) r_{0}+s r, t\right)}{i A(t)} \frac{r-r_{0}}{\alpha\left((1-s) r_{0}+s r\right)} d s .
$$

By hypothesis of the lemma on the gradient $w$, the second integral is equal to zero. Hence,

$$
v(r, t)=t \int_{0}^{1} \frac{w\left(r_{0}, s t\right)}{A(s t)} d s \equiv v(t) .
$$

It follows from Remark 12 that $v$ is constant, which implies $w \equiv 0$. Consequently, $\overline{T_{\alpha}} u=0$. Since $u$ is a real-valued function we also have $T_{\alpha} u=0$, and, therefore, $u$ is constant.

Proposition 14. Let $u(r, t)$ be a real-valued function. If $u \in C^{0}\left(\overline{A_{\epsilon}^{+}}\right)$is a solution of $P u=0$, then its $T_{\alpha}$-gradient $w$ satisfies $w \in C^{0}\left(A_{\epsilon}^{+} \cup\left(\{0\} \times \mathbb{S}^{1}\right)\right)$ and $w(0, t) \equiv 0$. Moreover, $u$ is constant along $\{0\} \times \mathbb{S}^{1}$.

Proof. If $u$ is constant, the lemma is trivial. Assume that $u$ is not constant, and let $w$ be its $T_{\alpha}$-gradient. Since $P$ is elliptic for $r \neq 0$, we need only to verify the continuity of $w$ up to $\{0\} \times \mathbb{S}^{1}$ and its vanishing there. We have that $w$ is a solution of $\mathcal{L} w=0$, and so it has a series representation

$$
w(r, t)=\sum_{\lambda_{j}^{ \pm} \geq 0}\left(c_{j}^{+} w_{j}^{+}(r, t)+c_{j}^{-} w_{j}^{-}(r, t)\right),
$$

where $w_{j}^{ \pm}(r, t)$ are the basic solutions defined in Theorem 5 .

Note that if in the above sum we have only $\lambda_{j}^{ \pm}>0$, then $w$ is flat along $\{0\} \times S^{1}$ (since each $w_{j}^{ \pm}$is flat). Suppose, by contradiction, that for some $j_{0}$ we have $\lambda_{j_{0}}=0$. Hence, we can rewrite $w$ in the form

$$
w(r, t)=v(t)+o\left(r^{n}\right), \quad \forall n \geq 1, \quad \text { where } \quad v \neq \equiv .
$$


It follows from Lemma 13 that $\operatorname{Re}\left(\frac{w_{j}(r, t)}{i A(t)}\right) \not \equiv 0$ for all $j$ and, consequently, $\operatorname{Re}\left(\frac{v(t)}{i A(t)}\right) \not \equiv 0$. Let $t_{0} \in \mathbb{R}$ such that $\operatorname{Re}\left(\frac{v\left(t_{0}\right)}{i A\left(t_{0}\right)}\right) \neq 0$. Let $r_{0}<R$ be fixed. We have

$$
u\left(r, t_{0}\right)-u\left(r_{0}, t_{0}\right)=\int_{0}^{1} \operatorname{Re}\left(\frac{v\left(t_{0}\right)}{i A\left(t_{0}\right)}\right) \frac{r-r_{0}}{\alpha\left((1-s) r_{0}+s r\right)} d s+o\left(r^{n}\right)
$$

for all $n \geq 1$. However,

$$
\operatorname{Re} \int_{0}^{1} \frac{v\left(t_{0}\right)}{i A\left(t_{0}\right)} \frac{r-r_{0}}{\alpha\left((1-s) r_{0}+s r\right)} d s=O(F(r)),
$$

which is a contradiction, since $u\left(r, t_{0}\right)-u\left(r_{0}, t_{0}\right)$ has limit as $r \rightarrow 0$ and $\lim _{r \rightarrow 0^{+}}|F(r)|$ $=\infty$. Therefore, $w$ is flat along $\{0\} \times \mathbb{S}^{1}$ and, consequently, $u(0, t)$ is constant.

In the next theorem we prove a series representation for solutions of the equation $P u=0$. First we define the functions $\phi_{j}^{ \pm}(t)$ by

$$
\phi_{j}^{ \pm}(t)=\operatorname{Re}\left(\frac{\psi_{j}^{ \pm}(t)}{\lambda_{j}^{ \pm} i A(t)}\right),
$$

where the $\psi_{j}^{ \pm}$are the components of the basic solution $w_{j}^{ \pm}$defined in Theorem 5 .

Theorem 15. Let $u(r, t)$ be a real-valued function. If $u \in C^{0}\left(\overline{A_{\epsilon}^{+}}\right)$is a solution of $P u=0$, then $u$ is constant on $\{0\} \times \mathbb{S}^{1}$ and it has the series expansion

$$
u(r, t)=u_{0}+\sum_{\lambda_{j}^{ \pm}>0}\left[u_{j}^{+} \exp \left(\lambda_{j}^{+} F(r)\right) \phi_{j}^{+}(t)+u_{j}^{-} \exp \left(\lambda_{j}^{-} F(r)\right) \phi_{j}^{-}(t)\right],
$$

where $u_{j}^{ \pm} \in \mathbb{R}$.

Proof. It follows from Proposition 14 that $u$ is constant on $\{0\} \times \mathbb{S}^{1}$. Hence, we have

$$
u(r, t)-u(0,0)=u(r, t)-u(0, t)=\operatorname{Re} \int_{0}^{1} \frac{w(r s, t)}{i A(t)} \frac{r d s}{\alpha(r s)},
$$

where $w$ is the $T_{\alpha}$-gradient of $u$. Since

$$
w(r, t)=\sum_{\lambda_{j}^{ \pm}>0}\left[c_{j}^{+} \exp \left(\lambda_{j}^{+} F(r)\right) \psi_{j}^{+}(t)+c_{j}^{-} \exp \left(\lambda_{j}^{-} F(r)\right) \psi_{j}^{-}(t)\right]
$$

we have

$$
\begin{array}{r}
u(r, t)=u(0,0)+\sum_{\lambda_{j}^{ \pm}>0}\left(\operatorname{Re}\left[\frac{c_{j}^{+} \psi_{j}^{+}(t)}{i A(t)}\right] \int_{0}^{1} \exp \left(\lambda_{j}^{+} F(r s)\right) \frac{r d s}{\alpha(r s)}\right. \\
\left.+\operatorname{Re}\left[\frac{c_{j}^{-} \psi_{j}^{-}(t)}{i A(t)}\right] \int_{0}^{1} \exp \left(\lambda_{j}^{-} F(r s)\right) \frac{r d s}{\alpha(r s)}\right) .
\end{array}
$$

Finally, representation (5.1) follows from this series and from

$$
\int_{0}^{1} \exp \left(\lambda_{j}^{ \pm} F(r s)\right) \frac{r d s}{\alpha(r s)}=\frac{\exp \left(\lambda_{j}^{ \pm} F(r)\right)}{\lambda_{j}^{ \pm}} .
$$




\section{ACKNOWLEDGMENTS}

The authors would like to thank the referee for comments and suggestions. Part of this work was done when the first author was visiting the Department of Mathematics at Florida International University. He is grateful and would like to thank the members of the host institution for the support they provided during the visit.

\section{REFERENCES}

1. H. Begehr, Complex analytic methods for partial differential equations. An introductory text, World Scientific Publishing Co., Inc., NJ (1994). MR1314196 (95m:35031)

2. A. Bergamasco, P. Cordaro, and J. Hounie, Global properties of a class of vector fields in the plane, J. Diff. Equ., 74 (1988), no. 2, 179-199. MR952894 (89h:58175)

3. A. Bergamasco, P. Cordaro, and G. Petronhilo, Global solvability for a class of complex vector fields on the two-torus, Comm. Partial Diff. Equ., 29 (2004), no. 5-6, 785-819. MR.2059148 (2005k:35034)

4. S. Berhanu, P. Cordaro, and J. Hounie, An introduction to involutive structures, New Math. Mono., 6, Cambridge University Press, Cambridge (2008). MR2397326 (2009b:32048)

5. P. L. Dattori da Silva, Nonexistence of global solutions for a class of complex vector fields on two-torus, J. Math. Anal. Appl., 351 (2009), no. 2, 543-555. MR2473960 (2009m:35038)

6. P. L. Dattori da Silva, $C^{k}$-solvability near the characteristic set for a class of planar complex vector fields of infinite type, Ann. Mat. Pura Appl., 189 (2010), 403-413. MR2657417

7. A. Meziani, Representation of solutions of planar elliptic vector fields with degeneracies, Geometric analysis of PDE and several complex variables, Contemp. Math., 368, 357-370, Amer. Math. Soc., Providence, RI (2005). MR2127042 (2005m:35032)

8. A. Meziani, Representation of solutions of a singular CR equation in the plane, Complex Var. Elliptic Equ., 53 (2008), no. 12, 1111-1130. MR2467386 (2009j:30105)

9. A. Meziani, Properties of solutions of a planar second-order elliptic equation with a singularity, Complex Var. Elliptic Equ., 54 (2009), no. 7, 677-688. MR2538058

10. A. Meziani, On first and second order planar elliptic equations with degeneracies, to appear in Memoirs of the AMS (see also arXiv:0910.0539v1).

11. F. Treves, Remarks about certain first-order linear PDE in two variables, Comm. Partial Differential Equations, 5 (1980), no. 4, 381-425. MR567779 (83e:35033)

12. F. Treves, Hypo-analytic structures. Local theory, Princeton Mathematical Series, 40, Princeton University Press, Princeton, NJ (1992). MR1200459 (94e:35014)

13. I. V. Vekua, Generalized Analytic Functions, Pergamon Press, Oxford (1962). MR0150320 $(27: 321)$

Departamento de Matemática, Instituto de Ciências Matemáticas e de Computação, Universidade de São Paulo, Caixa Postal 668, São Carlos, SP, 13560-970 Brazil

E-mail address: dattori@icmc.usp.br

Department of Mathematics, Florida International University, Miami, Florida 33199

E-mail address: meziani@fiu.edu 